\title{
Asynchronous telemedicine applications in rehabilitation of acquired speech-language disorders in neurologic patients
}

This article was published in the following Dove Press journal:

Smart Homecare Technology and TeleHealth

9 February 2015

Number of times this article has been viewed

\author{
Lilian Beijer' \\ Toni Rietveld ${ }^{2}$ \\ 'Sint Maartenskliniek, ${ }^{2}$ Centre \\ of Language Studies, Radboud \\ University of Nijmegen, Nijmegen, \\ The Netherlands
}

Correspondence: Lilian Beijer Sint Maartenskliniek, PO Box 90II, 6500 GM, Nijmegen, The Netherlands Email I.beijer@maartenskliniek.nl

\begin{abstract}
In this time of an aging population in the Western world and a concomitant need for cost reduction, there is an obvious need for innovative health care delivery. One of the consequences is that a growing number of telemedicine applications are emerging in different health care domains. Also in the area of speech-language (SL) disorders, particularly neurogenic disorders, telemedicine is rapidly gaining interest. In this paper, we place applications for neurogenic SL disorders in a telemedicine taxonomy in order to establish common features along the dimensions of functionality, application, and technology, and their components. Thus, we aim at identifying common features in a wide variety of telemedicine applications and to establish common interests of stakeholders in health care for classified groups of telemedicine applications. This may facilitate decision-making with regard to expansion of innovative products, and give directions to measures needed for upscaling and structural embedding of feasible and effective SL telemedicine applications in health care. Common interests of stakeholders in health care, established using telemedicine taxonomy, is a key factor in decision-making with regard to which telemedicine applications should be given priority for genuine utilization. Priorities of health care institutions, patients, and reimbursement companies are also leading for researchers aiming at solid scientific evidence for the beneficial effects of target applications. That is, although research results tend to indicate the potential of telemedicine in the area of SL pathology, the alleged benefits of most applications have not been confirmed according to the accepted standards for clinical outcome testing as yet. Methodologic obstacles and the lack of adequate speech materials and suitable outcome measures for efficacy and effectiveness testing partially account for this. From the perspective of scientific evidence, the benefits of asynchronous SL telemedicine applications concern data storage and data analyses. To facilitate implementation of telemedicine, there is a call for development of information and communication technology infrastructures that allow feasible applications which meet requirements with regard to licensure and medical privacy laws. For applications with evidence for beneficial effects, we are challenged to develop new business models that apply to a new health care environment.
\end{abstract}

Keywords: telemedicine, speech, language, disorders, rehabilitation

\section{Introduction}

There are many speech-language (SL) disorders, ranging from stuttering and voice disorders to developmental apraxia, dysarthria, and aphasia. Treatment protocols and treatment effects vary to a large extent; evidence for specific treatments is not always available, and treatment is often age-specific. Apart from the content of treatment, the modes of health care delivery increasingly vary due to developments in information and communication technology. Based on these developments, telemedicine applications, allowing remote interventions in SL pathology, are gaining interest. 
Telemedicine in general may overcome barriers with regard to access to services caused by distance or impaired mobility. ${ }^{1}$ In addition, the possibility of remote service delivery may be a solution to the threatening disbalance between the need for and availability of health care delivery in the years to come. That is, growing numbers of aging patients with chronic diseases, including neurologic conditions, are looking for high-quality health care in times of limited financial and therapeutic resources. ${ }^{2}$ For this reason, in the field of SL pathology, telemedicine applications may be particularly interesting for patients with SL disorders due to acquired neurologic diseases, such as stroke or Parkinson's disease (PD).

The American Speech-Language-Hearing Association is the professional, scientific, and credentialing association for more than 173,000 SL pathologists, audiologists, and SL and hearing scientists in the USA and elsewhere. The position of the American Speech-Language-Hearing Association is that telehealth is an appropriate model of service delivery for the SL pathology profession. Indeed, SL pathologists are becoming increasingly aware of the potential of telemedicine applications and are being challenged to adopt these new technologies in clinical practice. ${ }^{3}$ Although the technologies allowing telemedicine applications are promising for innovations in this field, we should take note of the following two issues. First, the use of telepractice does not remove any existing responsibilities in delivering services. Health care delivery by telemedicine technologies should meet the same requirements as those for traditional service delivery from the perspectives of quality of care and health care ethics. ${ }^{3}$ Second, enthusiasm for telemedicine on the part of policymakers and health officials has not always been matched by uptake and utilization in practice. It is therefore crucial to identify barriers to and facilitators of implementation. ${ }^{4}$

In this paper, we discuss applications for remote treatment of patients with acquired SL pathology due to neurologic diseases in the context of a telemedicine taxonomy. This taxonomy may help to better understand the implications for stakeholders in health care with regard to expansion of these innovative products for SL pathology. ${ }^{5}$ We specifically focus on asynchronous telemedicine applications for dysarthria (ie, distorted speech production due to diminished force and coordination of speech muscles) ${ }^{6}$ and aphasia (ie, distorted language understanding and production). ${ }^{7}$

\section{Telemedicine taxonomy}

We adopt the approach of Bashshur et $\mathrm{al},{ }^{5}$ who provide a clear and concise multidimensional taxonomy for telemedicine. This taxonomy does justice to the variety of telemedicine, and helps to identify the precise nature of a specific application. Thus, the confusion often caused by interchangeable use of terms like "telehealth", "telemedicine", "telecare", and "telepractice" can be avoided.

The taxonomy comprises two levels. The first level is grouped into three dimensions. Based on this first level, each telecare application can be classified according to functionality, specific applications, and technologic configurations. Subsequently, the second classification level allows grouping according to the components, as described below.

\section{Functionality}

The functionality dimension refers to functions that are performed and allows classification into the aspects of the medical care process. Activities such as prevention, diagnosis, treatment, follow-up, and rehabilitation are grouped into the four following components:

- Consultation, eg, prevention

- Diagnosis, eg, assessment

- Monitoring, eg, rehabilitation in the patient's home environment or telemetry for chronic patients with, for instance, diabetes

- Mentoring, eg, remote guidance of one specialist to another.

\section{Specific applications}

This dimension includes processes of care across basic (para) medical specialties, and subspecializations based on disease entities, sites of care, and treatment modalities:

- Medical specialty, eg, neurology, rehabilitation medicine

- Disease, eg, stroke, PD

- Site of care, eg, home, outpatient center

- Treatment (modality), eg, SL therapy, physiotherapy.

\section{Technologic configurations}

- Network, ie, virtual private network, open Internet, social networks

- Connectivity, ie, wired or wireless

- Synchronicity, ie, synchronous or asynchronous.

Synchronous services are conducted with interactive audio and video connection in real time (ie, videoconferencing). They create an in-person experience similar to that achieved in a traditional encounter. Synchronous services may connect a client/patient or group of clients/patients with a clinician, or they may include consultation between a clinician and a specialist. In asynchronous services, images or data are captured and transmitted (ie, stored and forwarded) for viewing 
or interpretation by a professional. Examples include transmission of voice clips, audiology testing results, or outcomes of independent client/patient practice.

\section{Asynchronous telemedicine applications for acquired neurogenic SL disorders}

Given the demand for equal quality of telemedicine compared with traditional health care services, there is an obvious need to prove the positive effects of telemedicine. This calls for data storage and data analyses, which fits with asynchronous technology. For this reason, in this paper on telemedicine in the rehabilitation of acquired neurogenic SL disorders, we focus on applications based on asynchronous technology.

We first give a short characterization of aphasia and dysarthria, being the most frequently occurring SL disorders after acquired neurologic diseases, and then review the most important developments with regard to telemedicine in this field. Finally, we discuss the challenges for implementation in (para) medical care.

Aphasia and dysarthria are the most common SL disorders resulting from neurologic diseases, such as Parkinson's disease (PD) stroke or traumatic brain injury.

Aphasia is a communication disorder that is usually acquired in adulthood and has to be distinguished from a (motor) speech disorder like dysarthria. The term "aphasia" encompasses a number of disturbances in the decoding of language and speech, in the generation of language, or both. Sometimes there is comorbidity, in the sense that both language production and speech production are affected. ${ }^{8}$ The ultimate goal of aphasia therapy is to achieve a level of functional communication that enables the person with aphasia to participate in society. However, in many therapies, the focus is quite restricted and primarily aims at facilitating word finding. 7,9

Unlike aphasia, dysarthria is not based on a language problem and has been defined as "a neurologic motor speech impairment that is characterized by slow, weak, imprecise, and/or uncoordinated movements of the speech musculature". ${ }^{10}$ Such impairment can result from damage to the central or peripheral nervous system. ${ }^{6}$

Both aphasia and dysarthria occur quite frequently after acquired neurologic diseases. The incidence of aphasia after stroke is estimated to be one third among those who survive. ${ }^{7}$ Dysarthria after stroke occurs in about $50 \%$ of cases, ${ }^{11}$ and the reported incidence of dysarthria in PD varies from 50\% to $90 \%$. With these considerable incidences in mind, it is obvious that, in a time of limited therapeutic and financial resources, the potential benefits of telemedicine for aphasic and dysarthric patients are appealing. The possibility to intensify training, independent of the physical presence of a speech therapist, is considered a main advantage since there is ample evidence for the beneficial effects of intensive training on communication rehabilitation. ${ }^{12}$ Apart from intensifying training, telemedicine also allows (independent) prolongation of training after discharge from therapy, with alleged positive effects on communication. With regard to aphasia, there is evidence that, although most recovery takes place in the first 6 months after stroke, people can continue to improve their language skills for several years. ${ }^{13}$ Also, for people with longstanding dysarthria resulting from stroke or $\mathrm{PD}$, there is evidence that their speech can still improve with treatment. ${ }^{6,14}$

Below we review developments in telemedicine applications for aphasia and dysarthria.

\section{Telemedicine in aphasia}

The development of computer programs for the training of aphasic patients started some 15 years ago, as it makes intensive therapy possible. ${ }^{15}$ In most programs, a mixture of text, graphics, and audio stimuli is presented. ${ }^{16}$ It is generally recognized that the presentation of visual stimuli enhances the effects of therapy in aphasic patients, as it relieves working memory and provides training of this important part of the language processing system. ${ }^{17,18}$ Providing immediate feedback (instead of delayed feedback) is also known to enhance the effects of therapy. ${ }^{19}$

In telemedicine applications for aphasia, synchronous and asynchronous technology tends to be combined by using hybrid technology systems. Thus, the main advantages of synchronous (ie, real-time interaction by videoconferencing) and asynchronous (ie, intensive, independent training, storage, and analyses of audio and video data) technology can be combined. ${ }^{20,21}$ For example, an aphasic patient is provided with a structured language intervention by asynchronous technology, while an SL pathologist is enabled to monitor real time by videoconferencing. Also, the possibility of only asynchronous language training is possible, with an SL pathology monitor or analyze the results asynchronously. An example is eREST (REduced Syntax Therapy), in which aphasic speakers with chronic agrammatism are stimulated to use elliptical constructions, and progress is assessed by a therapist. ${ }^{22}$

Although the above-mentioned teleapplications enable efficient, intensive, and individualized management of aphasia, the language training tends to be confined to a 
highly structured therapy practice context. Some telemedicine applications aim at an even more restricted goal: stimulating word finding by providing pictures and alternative responses, with or without automatic speech recognition. However, since recovery of functional communication in daily life is most beneficial for aphasic patients, virtual training environments (VTEs) for aphasia therapy offer challenging possibilities and are rapidly gaining interest. A VTE is an artificial environment that is created with software and is presented to the user in such a way that it is accepted as a real environment. In the case of aphasia therapy, a real environment is simulated for language training. A VTE allows patients to participate in social exchanges using a virtual character or "avatar" to represent themselves. Thus, they are allowed to develop communication skills and recreate a social environment to practice those skills. VTEs also have the potential to prevent social isolation since they create opportunities to create virtual encounters and may build up confidence for real-life social experiences. It is obvious that compliance with therapy may be enhanced by virtual training. Notwithstanding these potential applications, there are some technologic challenges with regard to the user interface required for aphasic patients to engage in VTEs. We will discuss these in some more detail.

Given the potential applications described above, VTEs for aphasia therapy should present all features that are characteristic of natural conversation, being visual presentation of a familiar environment and objects (eg, a shop, a home, objects of discourse) and autonomous agents that understand a patient's speech, and can react appropriately, both in speech and by visual and oral back channeling. The interaction between agents and a patient is especially challenging, since this requires complex technologic interaction.

A VTE requires interaction between the patient and two autonomous agents (a virtual therapist and a virtual character with whom the patient must communicate). The therapist agent will guide the tasks of the patient (eg, offering instructions on how to proceed with the training). The second agent will engage in task-relevant communication with the patient. The virtual agents fit in a recent line of research on virtual characters, sometimes also referred to as "embodied conversational agents", 23 "life-like characters", ${ }^{24}$ or "virtual humans". ${ }^{25}$ Technically, such characters can be seen as an integration of SL technology with computer graphics and artificial intelligence. SL technology is required to automatically process a user's input (ie, automatic speech recognition and natural language understanding), and to automatically produce the responses of the virtual character (ie, using natural language generation and speech synthesis). Computer graphics are needed to visualize the virtual characters and to animate their behavior. In recent years, substantial progress has been made in integrating the various "enabling technologies" for such virtual characters, ${ }^{26,27}$ and various applications have been developed. Artificial intelligence is needed to enable the virtual characters to emulate the communicative behavior that patients would expect from real persons.

Recent research has shown that it is possible to develop artificial intelligence and dialogue management for conducting natural conversations with a virtual character. ${ }^{28}$ Rapid developments in Semantic Web technology (SWT) are expected to accelerate patients' dynamic communicative interactions in VTEs which are materialized in increasingly varied forms. SWT is defined as “... an extension of the current Web in which information is given well-defined meaning, better enabling computers and people to work in cooperation". Once the Web has a mechanism for defining semantics about resources and links, then the possibility arises for automatic processing of the Web by software agents, rather than mediation by people. ${ }^{29,30}$ For example, in the case of VTE for aphasic patients, SWT would make it possible to generate target SL output on the basis of visual, auditory, or lexical features of objects or situations, based on semantic relationships with a possible target. However, SWT is not restricted to applications that are directly associated with the Web.

With regard to the automatic speech recognition used in VTEs for aphasic patients, there remain some challenges to be addressed. For example, while single-word recognition is not a difficult task for automatic speech recognition, the recognition of content units, which can vary both in length and wording, is difficult. It may not be robust enough for applications with patients, especially in the case of aphasia with comorbidity (dysarthria, apraxia of speech). That is why one can expect, in the near future, the development and application of more procedures that focus on word retrieval supported by sophisticated visual cues and scoring protocols. For a good example of such an approach, still without automatic speech recognition, we refer to the system described by Palmer et al. ${ }^{7}$ They showed that computer therapy can be beneficial for word finding, but less so for people with longstanding aphasia. The complicating factor in this well designed study is, however, the definition of "usual care" assigned to their control group. Usual care may vary from country to country, and from one clinical setting to another. 


\section{Telemedicine in dysarthria}

Just like in aphasic patients, Palmer et al found evidence that patients with chronic dysarthria benefit to the same extent from (off-line) computerized therapy as from traditional face-to-face therapy. ${ }^{6}$ Given the indications for the beneficial effects of intensive speech training after stroke in chronic neurologic patients, ${ }^{11,14}$ the potential applications of telemedicine in dysarthria are obvious. ${ }^{12}$

Most studies reported on telemedicine for dysarthria concern videoconferencing and are primarily aimed at speech training in patients with $\mathrm{PD}^{31}$ according to the LSVT (Lee Silverman Voice Treatment), which aims at increased vocal loudness. ${ }^{32}$ Although this telemedicine application seems promising, a disadvantage obviously lies in the synchronous technology, demanding the SL pathologist's presence during treatment sessions.

An asynchronous application for Web-based speech training (e-learning-based speech therapy) allowing patients with dysarthria to train their speech in their home environment has been developed by Beijer et al. ${ }^{33}$ They concluded that this telemedicine application allows intensive, tailor-made speech training in the patient's home environment as well as remote monitoring by SL pathologists. ${ }^{14}$

Dysarthric patients tended to appreciate the possibility of Web-based training, but usability should be adapted to the needs of this specific user group. Clinical outcomes research according to general standards should be conducted to support the alleged beneficial effects of Web-based speech training on intelligibility. ${ }^{34} \mathrm{~A}$ key benefit of an asynchronous store-and-forward application like e-learning-based speech therapy is the possibility of generating a Web-based database of dysarthric speech for therapeutic and research purposes. ${ }^{35}$ One of the research goals concerns improving automatic speech recognition of dysarthric speech.

Like for aphasia, there is a growing interest in VTEs for dysarthria. That is, due to the repetitive nature of regular speech exercises (eg, drills) for dysarthric speech, compliance with therapy is often lacking. This calls for motivating elements to improve compliance with speech therapy in patients who practice in their home environment. As in aphasia therapy, a VTE for dysarthria therapy should also represent features for natural conversation. In addition, automatic recognition of dysarthric speech should be developed to establish automatic feedback. This is a particularly challenging task for dysarthria, due to the variability of speech between and within dysarthric speakers. ${ }^{34}$ In the Netherlands, an interactive game for speech training in dysarthric patients is currently being developed in the CHASING (CHAllenging
Speech training In Neurological patients by interactive Gaming) project. ${ }^{36}$

\section{Challenges for implementation}

The rapid progress in information and communications technology and SL technology is clearly one of the reasons why thorough evaluation of their applications often lags behind their availability. In addition, lack of clarity regarding the specific goals and target groups of telemedicine applications may hamper decision-making with regard to upscaling. Therefore, the relevance of positioning newly developed applications in a telemedicine taxonomy and classifying them with regard to functionality, applications, and technology is obvious. Establishing common features in varying telemedicine applications may elucidate shared interests of stakeholders in health care, and enhance upscaling and utilization.

It is obvious that the emergence of telemedicine affects all stakeholders in the health care landscape (Figure 1). The technologic innovations induce adaptations in health care processes, along with the need for a cultural shift in both health care professionals and patients. In addition, government policy-makers and reimbursement companies have to reconsider measures to be taken to facilitate the adoption and upscaling of telemedicine.

\section{Research}

A key issue for accelerating telemedicine applications in the SL domain is the need for scientific evidence of their beneficial effects. Once clinical outcomes research has confirmed the advantages for the health care domain, policy-makers and reimbursement companies are liable to take measures for rapid integration and scaling of these telemedicine applications. With the importance of scientific evidence in mind, implementation of telemedicine in the domain of SL disorders constitutes a true challenge. That is, research on the effects of therapies for SL pathology is not easy for a number of reasons:

- As the heterogeneity of groups of neurologic patients with aphasia and/or dysarthria is often quite high, large numbers of patients are needed in order to reach an acceptable test power. However, this is not always possible (see under the next entry)..$^{38}$

- It is not always possible to find relatively large numbers of patients meeting specific requirements with regard to age, sex, severity of disease, time since onset, and socioeconomic status. This specifically holds for so-called small languages or minority languages. 


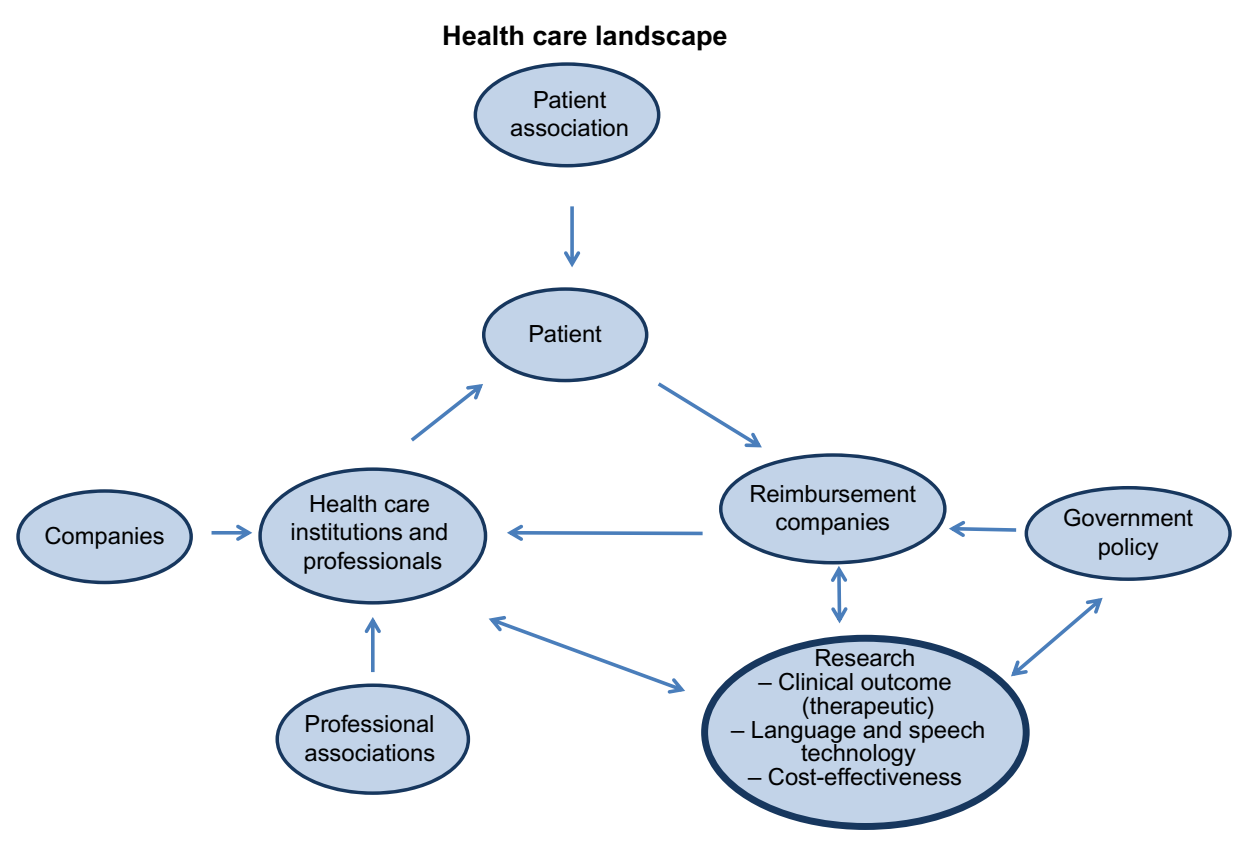

Figure I Stakeholders in the health care landscape.

Note: Adapted with permission from Janssen R, Hettinga M, Visser S, Menko R, Prins H, Krediet I, Haaker T Bodenstaff L. Innovation routes and evidence guidelines for e-health small and medium-sized enterprises. Towards feasible yet convincing evidence. International Journal on Advances in Life Sciences. 2013;5(3,4):188-203. ${ }^{37}$

- As the number of participants may be quite small, one cannot assume that a random selection of patients yields unbiased samples. That is why randomized controlled trials are often not feasible.

- Matching patients in treatment and control groups is often a difficult task, and is sometimes problematic from a statistical and methodologic point of view.

- It is difficult to avoid the well-known Hawthorne effect, especially when traditional and new computer-based approaches are compared. Participants might show a positive bias both in attitude and level of activity when an approach is offered that is new to them.

- It is difficult to establish suitable outcome measures that reflect genuine within-subject changes in the speech and language of neurologic patients. Below, we elaborate further on therapeutic outcome measures for aphasia and dysarthria.

\section{Outcome measures in aphasia therapy}

Relevant outcomes in aphasia therapy are largely treatmentdependent but also depend on the different types of aphasia and the time elapsed since the onset of the neurologic disease. The outcome measure of therapies aiming at facilitating word finding is relatively simple, ie, the number of words correctly associated with some picture, visual cue, or sound fragment. In some therapies, a relevant outcome is the realization of so-called content units, ie, parts of speech that are considered to be crucial for conveying a message. ${ }^{22}$ Detection of content units in the speech of persons with aphasia is not always easy, given that repetitions, nongrammatic expressions, and unexpected rephrasing can sometimes disturb the assessment procedures. Automatic detection of these units in spoken language will put high demands on the SL technology to be used. It illustrates the problems encountered in many telemedicine applications for SL pathology: the lack of robustness of SL technology, interfering with the necessity to automatically assess features of atypical speech and language which are also difficult to be assessed by trained therapists.

\section{Outcome measures in dysarthria therapy}

The ultimate outcome measures in dysarthria therapy are intelligibility and comprehensibility. Intelligibility is signaldriven and refers to speaker-dependent information (ie, the acoustic speech signal). Comprehensibility is the result of both speaker-dependent and speaker-independent information. Speaker-independent information can be controlled by the speaker (eg, gestures) but may also be determined by the situational context. ${ }^{39}$ Intelligibility measurement in particular is not an easy task since it is a true challenge to find ecologically valid speech materials that are also suitable for the outcome measures corresponding to intelligibility. ${ }^{34}$ That is, speech materials should reflect everyday conversation, but should 
also contain target elements that are sensitive to (acoustic) outcome measures corresponding to speech intelligibility. The quantity of speech assessment materials should also be taken into account. The enhancement of "environmental sensitivity" calls for larger amounts of speech materials, but the neurologic condition of dysarthric speakers does not allow long assessment sessions. Therefore, an adequate balance should be found between the need for enhanced sensitivity and the physical burden on dysarthric speakers. Apart from the type and speech materials for assessment, confounding variables such as ambient noise in home environments, should not affect measurements for intelligibility assessment.

\section{Government policy}

The World Health Organization challenges all governments to deliver better services to support health and well-being in order for people to live healthier and longer lives and to reduce inequalities in health care delivery. Health care delivery on the basis of telemedicine is one of the innovations that may contribute to the challenges of our aging population, which is characterized by increasingly demanding patients, a revolution of information, communication, and medical technology, and a call for care closer to home. It is important, particularly for the easily overlooked domain of SL therapy, to provide policy-makers with data and information to enhance well-informed decisions on telemedicine for SL disorders. ${ }^{11}$ From this perspective, the need for scientific evidence in telemedicine applications is obvious and governments are challenged to fund scientific research in this field. Priority of research (and hence, funding policy) may be established along the dimensions and components of the telemedicine taxonomy described in this paper. Telemedicine taxonomy may also support policy-making with regard to (state) licensure laws and medical information privacy laws. ${ }^{21}$

\section{Reimbursement companies}

It is clear that telemedicine for neurologic patients with SL disorders offers the potential to extend the continuum of care and improve the clinical outcomes for these patients. This is especially important from the perspective of insurance reimbursement challenges and shortened durations of hospital stay. ${ }^{40}$

Reimbursement companies base their decisions on costbenefit analyses during a time of rapidly changing health care environments. Therefore, they should play an active role in the development of new business models that apply to new technology-based health care environments. Rather than actual cash savings, models that aim at efficiency gains should be implemented. This calls for a broad orientation regarding the interests of all stakeholders in the health care landscape. At this point, the relevance of telemedicine arises again. That is, an overview of common interests of stakeholders may be supported by grouping telemedicine applications in the taxonomy. Establishment of applications with common functionalities, technologies, and applications may enhance the development of new business models that apply to changing health care environments.

\section{Health care institutions and patients (supported by patient and professional associations)}

Due to limited financial resources, health care institutions tend to discharge neurologic patients from clinical care early, and are therefore challenged to provide their services in a different manner. Obviously, from this perspective, telemedicine is appealing. It allows health care professionals to remotely monitor and mentor neurologic patients in order to intensify and prolong SL pathology services after clinical discharge. In addition, telemedicine applications based on asynchronous technology allow data storage and data analyses, which are important for being able to meet the increasing call for transparent and evidence-based health care. Data storage and analyses are clearly of common interest to researchers, health care institutions, and reimbursement companies.

SL pathologists and patients seem similarly anxious to adopt telemedicine technology for communication disorders resulting from neurologic disease. ${ }^{1,21}$ However, to lower the threshold for using telemedicine, its applications should be adapted to users' needs. ${ }^{41}$ Large user groups with common interests and needs may also be identified along the telemedicine taxonomy and serve as an argument for further development of telemedicine applications and new business models by reimbursement companies.

\section{Companies}

Companies delivering telemedicine technology should adapt their policies on product development to the common interests of health care institutions and patients. In addition, they should ensure the absence of interests that conflict with those of other stakeholders in order to successfully implement their innovative technology. ${ }^{37}$

\section{Future directions and conclusion}

The telemedicine taxonomy described in this paper provides the possibility for classification of different telemedicine developments along the dimensions of functionality, specific 


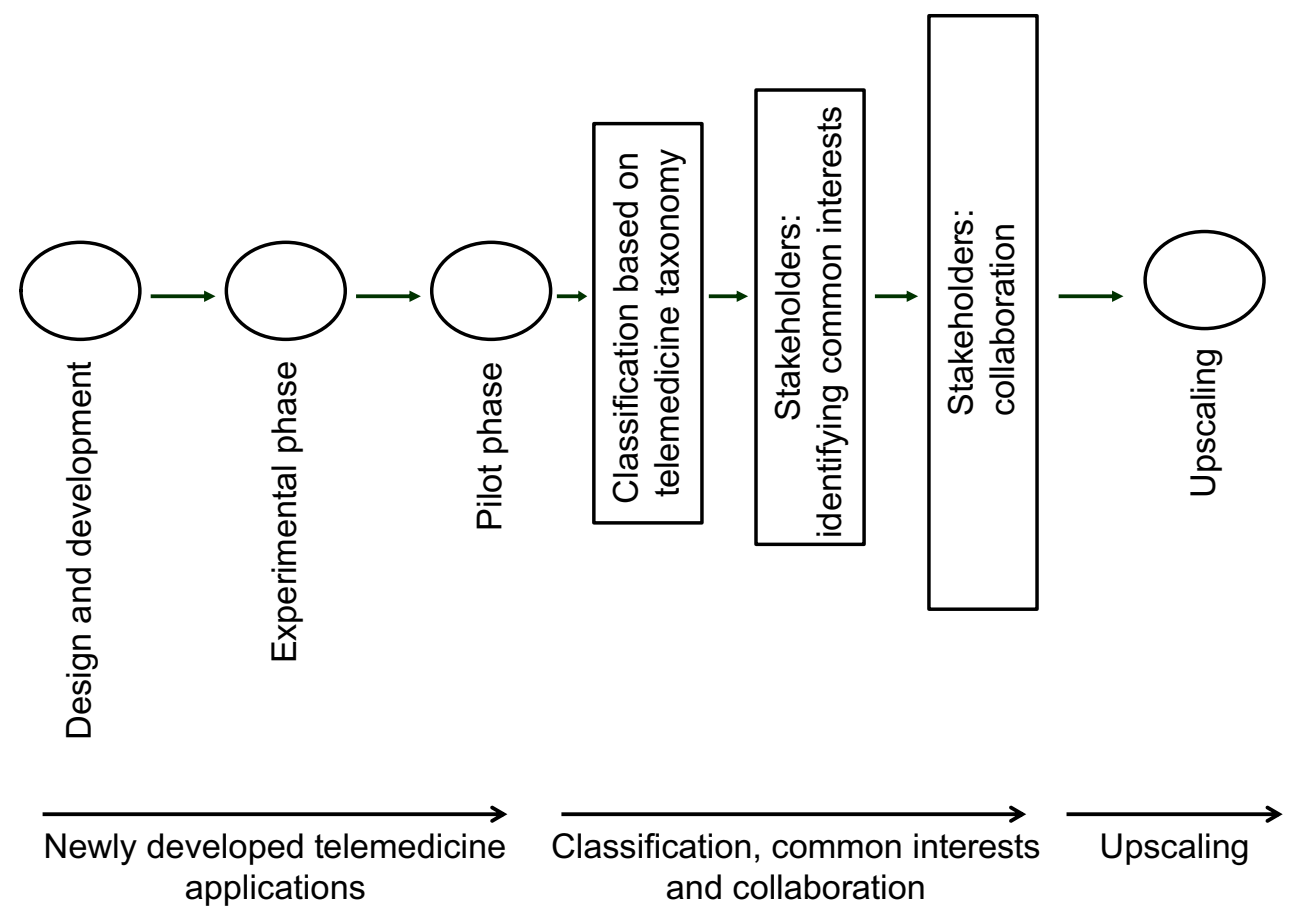

Figure 2 Stakeholders in health care identify common interests based on telemedicine taxonomy for the purpose of upscaling newly developed telemedicine applications.

applications, and technology, as well as their components. This classification provides an adequate basis upon which to group applications with common features, thus elucidating potential common interests (eg, asynchronous technology, remote assessment of patients with chronic conditions) for different stakeholders in health care. On the basis of stakeholders' common interests in specific grouped applications, they can estimate the number of potential users, costs, and research needs, and make decisions aimed at accelerating genuine utilization (Figure 2). Stakeholders may compare the magnitudes of common interests in different groups of telemedicine applications to prioritize upscaling. For example, dependent on the largest common interests, they may choose for either applications based on asynchronous technology for treatment of chronic neurologic patients with SL disorders in rehabilitation medicine, or for applications based on synchronous technology for stuttering patients who prefer web-based instead of face-to-face consults with their SL pathologist. The largest common interest of policy-makers, health care institutions, and reimbursement companies will be decisive. The research focus and policymaking of companies regarding product development will partially depend on the priorities of other stakeholders.

The taxonomy dimension of technologic configurations is of key importance since it is closely associated with the technologic feasibility, financial investments, licensure laws, and (medical) information privacy laws of telemedicine applications. A large common interest for the different stakeholders in the health care landscape therefore lies in the technologic infrastructure, and government policy-makers should take measures to stimulate the development of infrastructures that apply to licensure and (medical) information privacy laws, reimbursement companies aim at infrastructures that guarantee optimal cost-benefit ratios (eg, preferably asynchronous applications). This elucidates the central role of information and communication technology companies, with development and delivery of technologic infrastructure as their core business, and the vital importance of these companies carefully considering the common interests of the different stakeholders in technology for telemedicine in SL disorders.

In fact, successful upscaling of newly developed telemedicine applications requires collaboration of stakeholders to identify common interests and plan road maps for establishing genuine utilization in a new health care environment.

\section{Acknowledgment}

The authors thank Rosa van Bemmel for her assistance with the literature search.

\section{Disclosure}

The authors report no conflicts of interest in this work.

\section{References}

1. Mashima PA, Doarn CR. Overview of telehealth activities in speechlanguage pathology. Telemed J E Health. 2008;14:1101-1117. 
2. Beijer L. E-learning based speech therapy: exploring the potentials of e-health for dysarthric speakers. Unpublished dissertation. Nijmegen, The Netherlands: Radboud University; 2012.

3. American Speech-Language Hearing Association. Speech-language pathologists providing clinical services via telepractice, position statement 2005. Available from: http://www.asha.org/policy/PS2005-00116/. Accessed October 10, 2014.

4. Mair FS, May C, O’Donnell C, Finch T, Sullivan F, Murray E. Factors that promote or inhibit the implementation of e-health systems: an explanatory systematic review. Bull World Health Organ. 2012;90:357-364.

5. Bashshur R, Shannon G, Krupinsky E, Grigsby J. The taxonomy of telemedicine. Telemed J E Health. 2011;17:484-494.

6. Palmer R, Enderby P, Hawley M. Addressing the needs of speakers with longstanding dysarthria: computerized and traditional therapy compared. Int J Lang Commun Disord. 2007;42 Suppl 1:S61-S79.

7. Palmer R, Enderby P, Cooper C, et al. Computer therapy compared with usual care for people with long-standing aphasia poststroke. Stroke. 2012;43:1904-1911.

8. Whitworth A, Webster J, Howard D. Assessment and Intervention in Aphasia. Hove, UK: Psychology Press; 2005.

9. Agostini M, Garzon M, Benavides-Varela S, et al. Telerehabilitation in poststroke anomia. Biomed Res Int. 2014;2014:706909.

10. Yorkston KM. Treatment efficacy: dysarthria. JSpeech Hear Res. 1996;39: S46-S57.

11. Enderby P, Cantrell A, John A, Pickstone C, Fryer K, Palmer R. Guidance for providers of speech and language therapy services: dysarthrias. Asia Pac J Speech Lang Hear. 2010;13:171-190.

12. Winters JM, Winters JM. A telehome care model for optimizing rehabilitation outcomes. Telemed J E Health. 2004;10:200-202.

13. Meinzer M, Djunda D, Barthel G, Elbert T, Rockstroh B. Long term stability of improved language functions in chronic aphasia after constraint-induced aphasia therapy. Stroke. 2005;36:1462-1466.

14. Beijer LJ, Rietveld TC, Hoskam V, Geurts AC, de Swart BJ. Evaluating the feasibility and the potential efficacy of E-learning based speech therapy (EST) as a web application for speech training in dysarthric patients with Parkinson's disease: a case study. Telemed J E Health. 2010;16:732-738.

15. Hinckley J, Carr T. Comparing outcomes of intensive and non-intensive context-based aphasia treatment. Aphasiology. 2005;19:965-974.

16. van de Sandt-Koenderman MWME. High-tech AAC and aphasia: widening horizons? Aphasiology. 2004;18:245-264.

17. Nickels L, Best W. Therapy for naming disorders (Part II): specifics, surprises and suggestions. Aphasiology. 1996;10:109-136.

18. Wright HH, Shisler RJ. Working memory in aphasia: theory, measures, and clinical implications. Am J Speech Lang Pathol. 2005;14: 107-118.

19. Pedersen PM, Vinter K, Olsen TS. Improvement of oral naming by unsupervised computerized rehabilitation. Aphasiology. 2001;10:151-169.

20. Cherney LR, Kaye RC, Hitch RS, Onslow M. The best of both worlds: combining synchronous and asynchronous telepractice in the treatment of aphasia. Perspect Neurophysiol Neurogenic Speech Lang Disord. 2011;21:83-93.

21. Keck CS, Doarn CR. Telehealth technology applications in speechlanguage pathology. Telemed J E Health. 2014;20:653-659.

22. Ruiter MB, Kolk HHJ, Rietveld TC, Feddema I. Combining possibly reciprocally dependent linguistic parameters in the quantitative assessment of aphasic speakers' grammatical output. Aphasiology. 2013;27: 293-308.
23. Cassell J, Sullivan J, Prevost S, Churchill E. Embodied Converstational Agents. Cambridge, MA: MIT Press; 2000.

24. Prendinger H, Ishizuka M. Life-Like Characters: Tools, Affective Functions and Applications. Berlin, Germany: Springer; 2004.

25. Gratch J, Rickel J, Andre E, Badler N, Cassell J, Petajan E. Creating interactive virtual humans: some assembly required. 2002. Available from: http://www.media.mit.edu/gnl/publications/x4GEW.lo21.pdf. Accessed October 27, 2014

26. Lester J, Branting K, Mott B. Conversational agents. In: Singh MP, editor. Practical Handbook of Internet Computing. Baton Rouge: Chapman Hall and CRC Press; 2004.

27. Rickel JS, Marsella S, Gratch J, Hill R, Traum D, Swartout B. Towards a new generation of virtual humans for interactive experiences. IEEE Intelligent Systems. 2002;58:32-38.

28. Den Os EA, Boves L, Rossignol S, ten Bosch L, Vuurpij1 L. Conversational agent or direct manipulation in human-system interaction. Speech Commun. 2005;33:194-207.

29. Domingue J, Fensel D, Hendler J. Introduction to the Semantic Web technologies. In: Dominue J, Fensel D, Hendler J, editors. Handbook of Semantic Web Technologies. Berlin, Germany: Springer-Verlag; 2011.

30. Berners-Lee T, Hendler J, Lassila O. The semantic web. Sci Am. 2001;284:34-43

31. Cherney LR, van Vuuren S. Telerehabilitation, virtual therapists, and acquired neurologic speech and language disorders. Semin Speech Lang. 2012;33:243-257.

32. Ramig LO, Sapir S, Countryman S, et al. Intensive voice treatment (LSVT) for patients with Parkinson's disease: a 2 year follow-up. J Neurol Neurosurg Psychiatry. 2001;71:493-498.

33. Beijer LJ, Rietveld AC, van Beers MMA, et al. E-learning based speech therapy (EST): a web application for speech training. Telemed $J E$ Health. 2010;16:177-180.

34. Beijer LJ, Rietveld AC, Ruiter MB, Geurts ACH. Preparing an E-learning based speech therapy (EST) efficacy study: identifying suitable outcome measures to detect within-subject changes of speech intelligibility in dysarthric speakers. Clin Linguist Phon. July 15, 2014. [Epub ahead of print.]

35. Beijer L, Rietveld T. Potentials of telehealth devices for speech therapy in Parkinson's disease. In: Dushanova J, editor. Diagnostics and Rehabilitation of Parkinson's Disease, Book 6. InTech Open Access Publisher; 2011.

36. CHASING. CHAllenging Speech training In Neurological patients by interactive Gaming. Available from: http:/www.nwo.nl/onderzoeken-resultaten/onderzoeksprojecten/02/2300179302.html. Accessed October 10, 2014.

37. Janssen R, Hettinga M, Visser S, Menko R, Prins H, Krediet I, Haaker $\mathrm{T}$ Bodenstaff L. Innovation routes and evidence guidelines for e-health small and medium-sized enterprises. Towards feasible yet convincing evidence. International Journal on Advances in Life Sciences. 2013;5 $(3,4): 188-203$

38. Rietveld T, van Hout R. Statistics in Language Research: Analysis of Variance. Berlin, Germany: Mouton de Gruyter; 2005.

39. Yorkston KM, Strand EA, Kennedy MRT. Comprehensibility of dysarthric speech: implications for assessment and treatment planning. Am J Speech Lang Pathol. 1996;5:55-66.

40. Brennan D, Georgeadis A, Baron C. Telerehabilitation tools for the provision of remote speech-language treatment. Top Stroke Rehabil. 2002;8:71-78

41. Brennan DM, Barker LM. Human factors in the development and implementation of telerehabilitation systems. J Telemed Telecare. 2008;14: $55-58$. 


\section{Publish your work in this journal}

Smart Homecare Technology and TeleHealth is an international, peer-reviewed, open access online journal publishing original research, reviews, editorials and commentaries on the application of technology to support people and patients at home and in assisted living centers to optimize healthcare and management resources. Specific topics in the journal include: Development and application of devices within the home and embedded in appliances; Healthcare provider communication and education tools; and drug ordering and adherence. The manuscript management system is completely online and includes a very quick and fair peer-review system, which is all easy to use. Visit http://www.dovepress.com/ testimonials.php to read real quotes from published authors.

Submit your manuscript here: http://www.dovepress.com/smart-homecare-technology-and-telehealth-journal 